\section{The Chemical Nature of the Scrapie Agent}

By D. L. Mould. (Moredun Research Institute, Gilmerton, Edinburgh 9)

In any discussion as to the nature of the scrapie agent it is essential initially to consider the various phases of the disease. The agent can be transmitted naturally under suitable conditions between animals (Brotherston, Renwick, Stamp, Zlotnik \& Pattison, 1968). The route is unknown, but most probably ingestion and vectors other than airborne infection may be involved. After natural infection or experimental inoculation there is multiplication or replication of the agent over an extended timeperiod. During this period neurotropic degeneration is initiated by interaction between agent and nervous tissue producing malfunction in a chain of metabolic processes. Pathological change is not characteristic of virus infection and in non-neuronal tissue scrapie activity is not associated with any histopathological change. A complex interaction between these three aspects, governed by the original source of scrapie and the breed of sheep or strain of mouse involved, has been demonstrated in certain conditions to be highly dependent on genetic control (Dickinson, Meikle \& Fraser, 1968; Dickinson, Stamp, Renwick \& Rennie, 1968). Moreover, since only a few species are affected with scrapie and, with sheep, only a proportion of animals are fully susceptible, some inherited host factor appears to control further multiplication of the agent and tissue degeneration after infection.

There are enough points of similarity in the behaviour of two other diseases, kuru in man and encephalopathy in mink, to suggest that, although the causal agent is different and not interchangeable, the general pattern of development of the diseases is not unlike that of scrapie (Gajdusek, 1967). The experimental investigation of scrapie is much more advanced.

The disease condition has been investigated biochemically, firstly, from the viewpoint of the nature of the agent as determined by changes in biological response following chemical or physical treatment. Secondly, emphasis has been placed on the interaction of scrapie infective agent and host tissue, with consideration of possible mechanisms of multiplication involving nucleic acid synthesis, modification of metabolic enzyme activity related to histochemical changes in degenerating tissue, or clinical chemical studies in the affected animal (see Kimberlin, 1969). The elucidation of scrapie undoubtedly lies in linking these two approaches together in a determination of the sequence of events in the affected tissue. For example, although an early increase in synthesis of nuclear DNA has been demonstrated in scrapie-affected mice (Kimberlin
\& Hunter, 1967), washed nuclei are free of scrapie activity; an increase in the rate of synthesis of brain nuclear histones at a later stage in infection (Caspary \& Sewell, 1968) also does not appear to be related to any scrapie activity in separated nucleohistones. The response of the scrapie agent as present in brain from clinically affected mice to various physical and chemical agents has been reviewed by Gibbons \& Hunter (1967). The resistance to the effect of ionizing radiation and u.v. irradiation, heat, formalin and solvent action is of particular interest. However, substances that disturb secondary chemical structure do have a striking effect on the activity of the scrapie agent, e.g. strong acid or alkali, aqueous $90 \%$ phenol or $8 \mathrm{M}$-urea will inactivate and exposure to $0.01 \mathrm{M}$-periodate or strong salt solutions will result in a substantial loss of titre. After treatments such as fluorocarbon extraction or action of ficin the activity becomes more sensitive to subsequent heating. Before the rise in titre in the central nervous system there is an early rise in infectivity in the spleen and lymph nodes. This raised the possibility that the agent might be in a modified or changing form in these organs during the period of increasing titre. Recent investigation (D. L. Mould, unpublished work) has demonstrated a few alterations in infected spleens : for example, the activity is not sensitive to heat treatment after fluorocarbon extraction, and there is a decrease in titre after the action of trypsin. Because of the association of scrapie activity with cellular membrane fragments it is difficult to assess the effect of chemical action as disruption of a specific binding site for the scrapie agent producing a labile entity, or as complete breakdown of an integral structure carrying scrapie activity.

A slow-acting virus conception will explain the transmission and distribution of activity in host tissues, but irradiation data (Alper, Cramp, Haig \& Clarke, 1967) strongly suggest the complete absence of nucleic acid. Together with considerations of the size and chemical and physical properties of the scrapie agent the entity must be unlike any known virus in structure and dimension. Adams \& Caspary (1967) have proposed a resistant polysaccharide-coated nucleic acid core as a hypothetical model, but the nucleic acid problem remains. Observations with labelled DNA or polysaccharide precursors have shown changes from normal in the rates of uptake (or formation of breakdown products), but this is tangible evidence for metabolic changes in scrapie-affected tissue rather than for synthesis of the polysaccharide-nucleic acid virus proposed (Adams, Caspary \& Field, 1969). Isolation of polysaccharide or protein constituents from infected brain have shown no scrapie activity (Hunter, Gibbons, Kimberlin \& Millson, 1969; G. C. Millson \& G. D. 
Hunter, unpublished work). The sensitivity of tissue homogenates to urea, phenol and some proteolytic action suggests involvement of protein, but the reverse is suggested by the stability to heat and formalin. Similarly the sensitivity of scrapie to periodate is suggestive of polysaccharide involvement, but unfortunately methods of isolation that utilize phenol or urea inactivate.

The association of scrapie activity with plasma membrane and endoplasmic-reticulum fragments has been discussed by Hunter (1969). This substantiates hypotheses proposed by Gibbons \& Hunter (1967) and Adams \& Field (1968) that both the disease process and replication of the agent are located in a steric rearrangement of the component layers in a cellular membrane. Broadly considered as a protein-phospholipid doublelayered structure with attached oligosaccharide residues, this conception allows for the variable effects of chemical treatment. Variations in permeability in different cell populations can explain the pathological changes in brain tissue compared with other tissues and also the probable differences in scrapie replication rate in the various organs after scrapie inoculation. Formation of membrane in the presence of a foreign component might well require an induced enzyme system whose own synthesis would be under genetic control.
The mode of replication of a modified membrane structure is problematical, but the stage has often been reached when malfunction has been postulated in a cellular process whose normal function, structure and differentiated synthesis is, itself, as yet not fully understood.

Adams, D. H. \& Caspary, E. A. (1967). Brit. med.J. 3, 173. Adams, D. H., Caspary, E. A. \& Field, E. J. (1969). J.gen. Virol. 4, 89.

Adams, D. H. \& Field, E. J. (1968). Lancet, ii, 714.

Alper, T., Cramp, W. A., Haig, D. A. \& Clarke, M. C. (1967). Nature, Lond., 214, 764.

Brotherston, J. G., Renwick, C. C., Stamp, J. T., Zlotnik, I. \& Pattison, I. H. (1968). J. comp. Path. 78, 9.

Caspary, E. A. \& Sewell, F. M. (1968). Experientia, 24, 793.

Dickinson, A. G., Meikle, V. M. H. \& Fraser, H. (1968). J. comp. Path. 78, 293.

Dickinson, A. G., Stamp, J. T., Renwick, C. C. \& Rennie, J. C. (1968). J. comp. Path. 78, 313.

Gajdusek, D. C. (1967). New Engl. J. Med. 276, 392.

Gibbons, R. A. \& Hunter, G. D. (1967). Nature, Lond., 215, 1041.

Hunter, G. D. (1969). Biochem. J. (in the Press).

Hunter, G. D., Gibbons, R. A., Kimberlin, R. H. \& Millson, G. C. (1969). J. comp. Path. 79, 101.

Kimberlin, R. H. (1969). Biochem. J. (in the Press).

Kimberlin, R. H. \& Hunter, G. D. (1967). J. gen. Virol. 1, 115.

\section{COLLOQUIUM ON 'STRUCTURE, FUNCTION AND BIOSYNTHESIS OF SMALL RIBONUCLEIC ACID SPECIES'}

\section{Fractionation of Low-Molecular-Weight Ribo- nucleic Acid}

By U. E. Loening. (Department of Botany, University of Edinburgh)

The fractionation of RNA in the size range from t-RNA* to the smaller ribosomal RNA will be considered. In general, methods that fractionate according to the composition of the RNA have been used for t-RNA, and methods dependent on molecular weight have been used for the others. This is largely because of the present stage of knowledge of the heterogeneity of t-RNA and the need to prepare pure t-RNA fractions, and the state of ignorance of the significance or function of the other low-molecular-weight RNA components.

Examples will be given of the fractionation of t-RNA by countercurrent distribution, by methylated albumin-kieselguhr and reversed-phase chromatography, and by DEAE-cellulose, DEAESephadex and related columns. These methods

* Abbreviation: t-RNA, transfer RNA. have shown differences in the t-RNA distribution in different tissues and t-RNA unique to the mitochondria and chloroplasts. Rapidly labelled lowmolecular-weight RNA also shows fractions obtained by methylated albumin-kieselguhr chromatography that are distinct from the t-RNA and 5s RNA.

Fractionation by molecular weight has shown the existence of several types of RNA distinct from t-RNA. Column chromatography by methylated albumin-kieselguhr or by gel filtration, sedimentation, and gel electrophoresis will be compared. The gel-filtration methods depend almost entirely on molecular-weight differences and are not much influenced by composition or conformation. Gel electrophoresis gives the greatest resolution, but the column methods have the advantage that large amounts of RNA can be fractionated.

Examples of the gel-electrophoretic analysis of RNA will be shown to illustrate the following findings.

(a) Several homogeneous types of RNA other than t-RNA, 5s RNA and 7s RNA can be found. 